\title{
Perspektiven in der Onkologie
}

In die systemische Therapie des Mammakarzinoms kommt zunehmend Bewegung, ja Beschleunigung. Dies lässt sich sowohl in der adjuvanten als auch in der palliativen Behandlungssituation erkennen und findet sich sowohl im Bereich der Chemotherapie wie in der endokrinen Therapie. Klar erkennbar ist die Tendenz, die maximal wirksamen Substanzen wie Anthrazykline und Taxane vermehrt mit kurativer Absicht in die adjuvante Situation einzubringen. Diese Bestrebung wird unterstützt durch die Beobachtungen in der primär systemischen Therapiesituation moderner Studien, in denen der Einsatz der Taxane generell zu einer deutlichen Steigerung der Komplettremissionsrate (pCR) führt. Dies unterstützt das Konzept, die Taxane primär auch in der adjuvanten Therapie zum Einsatz zu bringen um die Überlebensraten weiter zu erhöhen. Bereits jetzt deutet die Datenlage darauf hin, dass vor allem Hochrisikopatientinnen von einer zusätzlichen taxanhaltigen adjuvanten Chemotherapie profitieren. Einen endgültigen Überblick über das Ausmaß des zusätzlichen Benefits durch Taxane werden wir dann erhalten, wenn die Ergebnisse der 5 weltweit laufenden Studien mit insgesamt etwa 20000 Patientinnen ausgewertet sein werden. Es sei hier darauf hingewiesen, dass für einige der genannten Substanzen und Kombinationen bislang keine Zulassung in bestimmten, vor allem in adjuvanten Therapiesegmenten besteht.

Die alte Streitfrage, ob hochwirksame Substanzen adjuvant in Kombination oder Sequenz verabreicht werden sollen, ist durch die elegante Studie von Citron et al. [J Clin Oncol 2003;21: 1431-1439] gelöst, bei der gezeigt wurde, dass die Ergebnisse gleichwertig sind. Aus den Daten geht allerdings auch eindeutig hervor, dass eine erhöhte Dosisdichte zu einer Verbesserung der Überlebensraten führt.

Auch in der adjuvanten endokrinen Therapie ist Bewegung. Die lange Zeit vernachlässigte adjuvante endokrine Therapie der jungen prämenopausalen Patientinnen mit Hormonrezeptor positivem Tumor nach adjuvanter Chemotherapie findet zunehmend Beachtung. Die konsequente Blockade der Ovarialfunktion in Kombination mit Tamoxifen verspricht weitere Verbesserung der Ergebnisse. In der Postmenopause werden die modernen Aromatasehemmer Einzug in die adjuvante en- dokrine Therapie nehmen. Die ersten Daten der ATAC-Studie zeigen auch hier weitergehende Verbesserungen gegenüber dem bisherigen Goldstandard Tamoxifen. Nach 5 Jahren Tamoxifen kann die Fortführung der adjuvanten Therapie mit Letrozol die Metastasierungsrate weiter absenken. Große Erwartungen werden in den adjuvanten Einsatz des humanisierten monoklonalen Antikörpers Trastuzumab (Herceptin ${ }^{\circledR}$ ) beim Her-2/neu exprimierenden primären Mammakarzinom gesetzt. Vier adjuvante Therapiestudien mit Trastuzumab unter Einschluss von insgesamt mehr als 10000 Patientinnen rekrutieren weltweit mit überdurchschnittlicher Geschwindigkeit und werden diese brennend interessierende und wichtige Frage bald beantworten können.

Auch beim metastasierten Mammakarzinom ist Fortschritt erkennbar. In der Hochrisikosituation kann mit aktiven Docetaxel $\left(\right.$ Taxotere ${ }^{\circledR}$ ) Kombinationen in der First-line-Therapie sogar eine Verlängerung der Überlebenszeit erreicht werden. Auch sequenzielle Monotherapien sind bei hoher Effektivität und reduzierter Toxizität erfolgreich. Vor allem beim Her2/neu exprimierenden metastasierten Mammakarzinom ist die Kombination von Trastuzumab mit Docetaxel, Capecitabin oder Vinorelbine hoch effektiv mit Remissionsraten bis zu $80 \%$ und gleichzeitig geringer Toxizität.

Der Blick in die Zukunft zeigt uns ein deutlich verbreitetes therapeutisches Spektrum auch dann, wenn Anthrazykline und Taxane im zunehmenden Maße vorab in der adjuvanten Therapie zum Einsatz kommen. Orale Chemotherapien wie Capecitabine oder liposomale Anthrazykline, die beide nicht zur Alopezie führen sowie wöchentliche Therapiemodalitäten gegebenenfalls in Kombination mit Trastuzumab bereichern unser Armentarium im Kampf gegen den metastasierten Brustkrebs. Der individualisierte, sequenzielle Einsatz der verschiedenen modernen Formen der endokrinen Therapie gefolgt vor hocheffektiven nebenwirkungsarmen Chemotherapieregimen bietet bereits jetzt deutlich verbesserte Ansätze der Behandlung in der Metastasierung. Vor dem Hintergrund der sich entwickelnden Möglichkeiten rückt das Ziel der Lebensverlängerung bei Langzeitremission und guter Lebensqualität immer mehr in den Vordergrund der Bemühungen.

Fritz Jänicke, Hamburg 